\title{
Spinal Manipulation and Dynamic Neuromuscular Stabilization Care for a 4-Year-0ld Patient with Agenesis of the Corpus Callosum
}

\author{
Michael Oppelt, Virginia Barber, Susan Larkin, Brennan Roberts \\ Palmer College of Chiropractic, Davenport, IA, USA \\ Email: oppelt_m@palmer.edu
}

How to cite this paper: Oppelt, M., Barber, V., Larkin, S. and Roberts, B. (2016) Spinal Manipulation and Dynamic Neuromuscular Stabilization Care for a 4-Year-Old Patient with Agenesis of the Corpus Callosum. Journal of Behavioral and Brain Science, 6 , 498-508.

http://dx.doi.org/10.4236/jbbs.2016.612045

Received: September 21, 2016

Accepted: October 31, 2016

Published: November 3, 2016

Copyright $\odot 2016$ by author and Scientific Research Publishing Inc. This work is licensed under the Creative Commons Attribution International License (CC BY 4.0).

http://creativecommons.org/licenses/by/4.0/

\begin{abstract}
The purpose of this case study is to describe the chiropractic care of a 4-year-old male patient with agenesis of the corpus callosum. Methods: Chiropractic care plan consisted of weekly appointments with the inclusion of at-home exercises coupled with academic intervention of physical and occupational therapies and assistive gait devices. Functional changes were monitored via objective clinic findings, independent clinical examination, and parental observation. Results: Agitated flexion contracted non-weight bearing child with gastrointestinal dysfunction and developmentally shunted growth responds to co-managed chiropractic care. Focus on aiding structural balance helped improve the weight bearing movement and mobility, physical calmness and contentment, emotional and verbal communication, as well as gastointestinal function. Discussion: This therapeutic approach decreased aberrant posture and enhanced quality of life. Conclusion: Chiropractic care in combination with academic intervention improved this child's postural abnormalities, attitude, and cognitive development warranting consideration in subsequent care investigation.
\end{abstract}

\section{Keywords}

Agenesis of Corpus Callosum (AgCC), Chiropractic, Dynamic Neuromuscular Stabilization (DNS), Individualized Educational Program (IEP)

\section{Introduction}

The corpus callosum connects both hemispheres of the cerebrum and is the largest white matter tract in the brain [1]. The corpus collosum's primary function is the transfer of sensory, motor, and cognitive information between hemispheres. 
Agenesis of the corpus callosum (AgCC) is a relatively common disorder. It is estimated to occur in at least $1 / 4000$ live births [2] with a symptomatic spectrum dependent on the extent of the dysgenesis. AgCC has been found in 3\% - 5\% of patients with development disorders [2], including Arnold Chiari malformation (cerebellar tonsil invagination), hydrocephalus, ventricular hypertrophy (including colpocephalyaka hypertrophy of the occipital horn), along with environment factors such as fetal alcohol syndrome [3].

There has not been a specific cause identified occurring within the $14^{\text {th }}$ and $18^{\text {th }}$ week of pregnancy that interrupts the normal development of the midline brain [4]. During normal corpus callosum development axons from one hemisphere navigate across the midline of the brain, directed by guidepost cells that secrete neurochemical cues, coordinating axonal growth via directional wiring to specific targets on the opposite hemisphere. Recent literature identified two neurochemical components that contributed to proper development of the corpus callosum. These components, tumor suppressor Nf2 and transcriptional coactivator Yap, require a synergistic concentration gradient [5]. When Nf2 concentration is depleted relative to Yap concentrations, it is ineffective at inhibiting Yap promotion of guidepost cell differentiation and monitoring Slit2 expression. Conversely, the natural up regulation of Yap (with normal Nf2) will cause the same effects as Nf2 depletion [5]. This theory is very promising and current, but remains a theory among many others attempting to identify AgCC causation.

Patients with AgCC will exhibit the condition in a variety of ways, which has led to symptom intervention treatment rather than attempts to influence the underlying neuroanatomy [6]. Many patients' AgCC will reach developmental milestones later in life compared to individuals with normal corpus callosum development. One study examined the cognitive milestone accomplishments relative to extent of AgCC dysgenesis as compared to the "normal" brain. It is determined that the degree of disability is directly related to the degree of dysgenesis [7]. AgCC developmental achievement represents cross cortical communication in the absence of the corpus callosum.

A fascinating aspect noted by Fernanda Tovar-Moll and his team identified the corpus callosum was the only white matter bundle to be partially missing or absent in patients with AgCC. Even in patients with colpocephaly (occipital horn hypertrophy), all posterior white matter bundles were found to be developed. Furthermore, studies show the development of Probst bundles are larger in AgCC individuals in comparison to the normal cerebrum, and exhibit connectivity to the different lobes of the brain ipsilaterally [8]. This implies that relatively normal cerebral white matter bundle development is present in a person with AgCC except the corpus callosum itself [8]. In fact this study concluded that subjects with callosaldysgenesis could transfer complex tactile information across hemispheres via posterior and anterior commissures. This is achieved by the cerebrum's neuroplasticity and its ability to adapt through cortical remapping of these commissures [8].

The following case study will focus on the chiropractic treatment of a 4-year-old male patient with agenesis of the corpus callosum and minor reference to dynamic 
neuromuscular stabilization treatment and school therapies.

A 4-year-old patient with agenesis of the corpus callosum presented to our chiropractic clinic. Initially Pub Med, Cochrane Library, Dyna Med, and ICL were investigated in order to gain a viable treatment plan or protocol in relation to the disorder. No functional information relative to chiropractic care or any other alternative medicine was uncovered for the AgCC condition during the search. There was very little information given in terms of treatment by traditional medical protocols as well. The lack of information associated with success of known treatment strategies provided motivation to initiate a chiropractic treatment protocol. The chiropractic care plan integrated the combination of rehabilitative therapy and co-management with other occupational specialists working with the patient through his school at the time.

The mother reported that her pregnancy was unremarkable until approximately one month before the anticipated birth date. Tests were administered prenatally due to the concern of hydrocephalus. The patient was born one month premature. She shared that her son was frank breech and her anomitic fluid levels were low which resulted in an emergency C-section. A MRI was performed the day after the birth, see Figure 1, and the radiologist determined the issue was not hydrocephalus but rather agenesis of the corpus callosum in combination with parallel configuration of the lateral ventricles with colpocephaly (occipital horn hypertrophy) and minor prominence of the temporal horns.

The patient weighed approximately $5 \mathrm{lbs}$. at birth. He was frequently agitated, colicky and crying. The mother shared her son had trouble latching during the first month of life, which was complicated by his constant flexed posture. The infant exhibited visual nystagmus and initially diagnosed with optic nerve atrophy. A follow up examination at
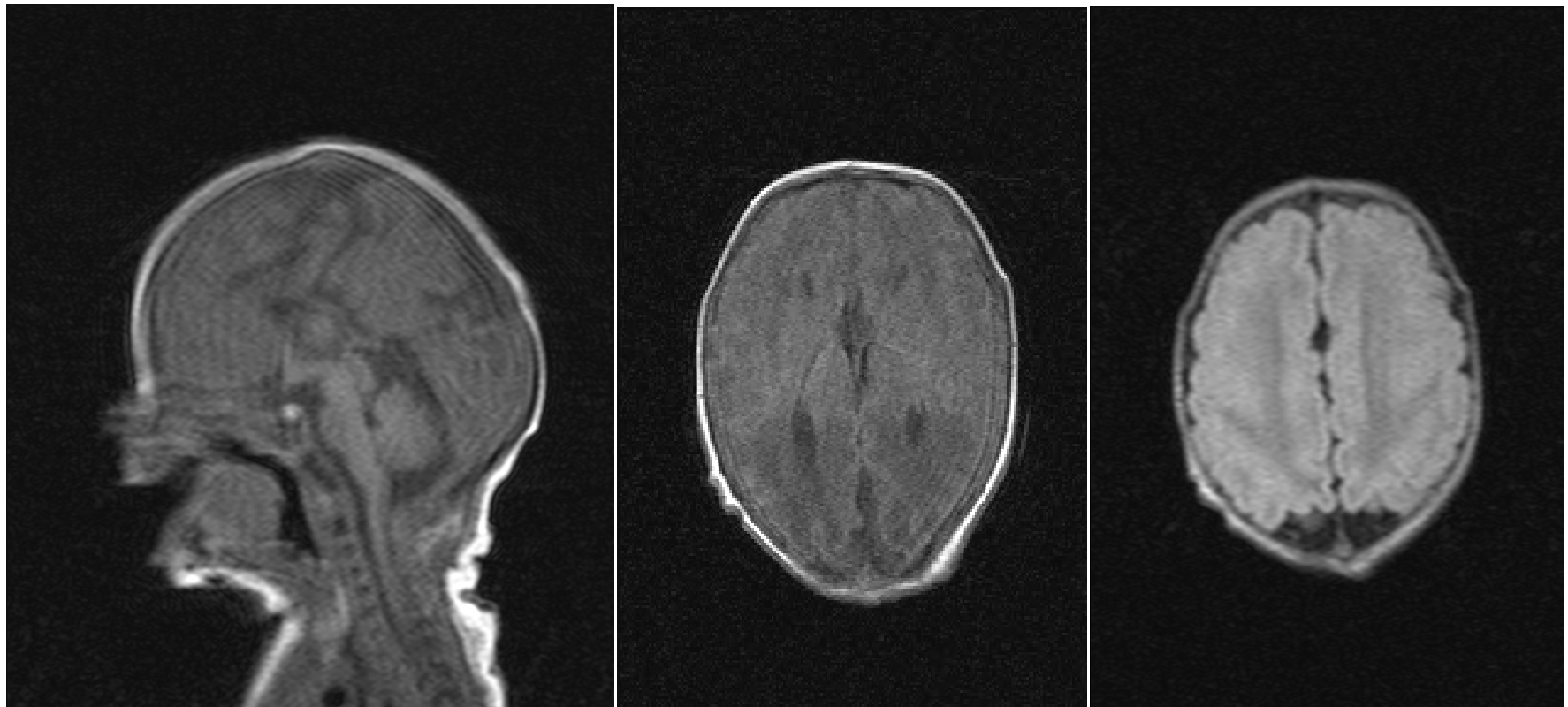

Figure 1. MRI showing opposing views of the patients' AgCC. Sagittal T1 (left), axial T1 (center), coronial T2 (right). 
three years of age determined there was no evidence of optic nerve atrophy. Genetic testing for possible causation of the presentation determined the onset of AgCC as idiopathic.

\section{Methods}

The four year old male patient's initial presentation was irritated, aggravated, and crying. The child did not, could not, functionally utilize his legs in a weight bearing position. The mother expressed that her boy did not seem "content." She stated that he was not yet toilet trained and had roughly eight wet/soiled diapers a day and was constantly "trying to go to the bathroom." The child's aggressive straining to pass a bowel movement frequently resulted in a rash. The mother indicated the patient tries to speak 3 to 4 words and responds to about 10 words but believes he can comprehend fairly well. The child currently participates in exercises with a physical therapist (PT), occupational therapist (OT), and speech therapist (ST) at school. The patient consistently responds peacefully to music.

The mother reports the patients current lifestyle habits consist of: going to school on a regular basis, working with teachers, PT's, OT's, and ST's at school and sleeping about 10 to 11 hours a night.

At the initial developmental assessment the patient was able to meet the following milestones: lift his head for several seconds, lift his head above body plane, turn his head toward an object, sit unsupported with his head steady, reach for objects, lift head while supine, sit alone in a tripod fashion, feed himself a cracker, crawl with legs flexed and crossed, smile responsively, begin to vocalize, smile and vocalize in response, turn his head toward sounds, smile spontaneously, babble, localize direction of sounds, drink from sipping cup, throw a ball, say specifically, "Dada"/"Mama". The patient keeps his right hand in a tightly flexed position. While seated or crawling the patient kept the lower legs crossed.

The patient was not able to meet the following milestones: climb, play with several children, button large buttons, comprehend his own age and sex, stand independently, walk, walk with assistance, climb stairs, climb stairs with assistance, recognize three colors, speak 2 - 3 word sentences, handle a spoon or fork functionally, use intelligible words beyond mama and dada, pedal tricycle, kick ball, wash hands, and wave bye-bye.

The initial physical evaluation documented the patient was 47 inches in height and 40 pounds in weight. The patient's posture was a flexed seated position with flexed, rigid legs and a constantly flexed right hand. The following aspects of the exam could not be performed due to patient tolerance: blood pressure, gait, internal ear exam, orthopedic exams, percussion, and many neurological exams. The limited examination revealed a normal temperature (98.3 degrees Fahrenheit), respiration (20 breaths per minute) and pulse ( $84 \mathrm{bpm})$. The external eye exam exhibited left eye lateral strabismus and patient unable to perform near point reflex, cardinal planes of gaze, and internal eye exam. However, patient's mother reports that previous optometrist reported previous diagnosis of optic atrophy was no longer valid and the optic nerve appears heal- 
thy and functioning well. Testing of the Vagus nerve, hypoglossal nerve, and glossopharyngeal nerve all yielded no abnormalities detected. Respiratory evaluation revealed that the patient sounded congested, but the mother reports that this was a recent development. Carotid pulses, radial/brachial pulses, femoral/popliteal pulses, and auscultation all yielded no abnormalities detected. Abdominal exam revealed scratches on the skin, minimal peristaltic sounds, and dull lower left quadrant percussion sounds. Patient also had cranial asymmetry with a flattened occipital bone and facial asymmetry. The palpation findings included sacrotuberous tension increased on the right decreased sacral motion, suboccipital muscle hypertonicity with the head in an extended position and decreased occipital fluid motion bilaterally. Thoracolumbar paraspinal myohypertonicty with fixed extension postural dysfunction. Most of the subjective information involving pain and symptoms were not obtainable due to the patient's communication deficits secondary to his AgCC disorder.

The goals outlined for the patient consisted of an objective increase in child physical contentment and happiness, decrease time in developmental milestones, and aim to have the patient ambulatory. The initial treatment plan consisted high velocity low amplitude chiropractic adjustment technique.

Treatment began with conservative Chiropractic care of full spine adjusting on a twice-weekly bases (Figure 2). At the $13^{\text {th }}$ week of treatment, the patient was referred to clinic's rehabilitative facility for evaluation and second opinion regarding rehabilitative inclusion into his care plan. The rehabilitation clinician conclude the child had poor scapular stability and excessive hip flexion with abduction noted bilaterally. The right wrist was in a flexed contracted position with the fingers making a fist and the left hand in an extended ulnar deviated position. The patient exhibited scapular elevation and

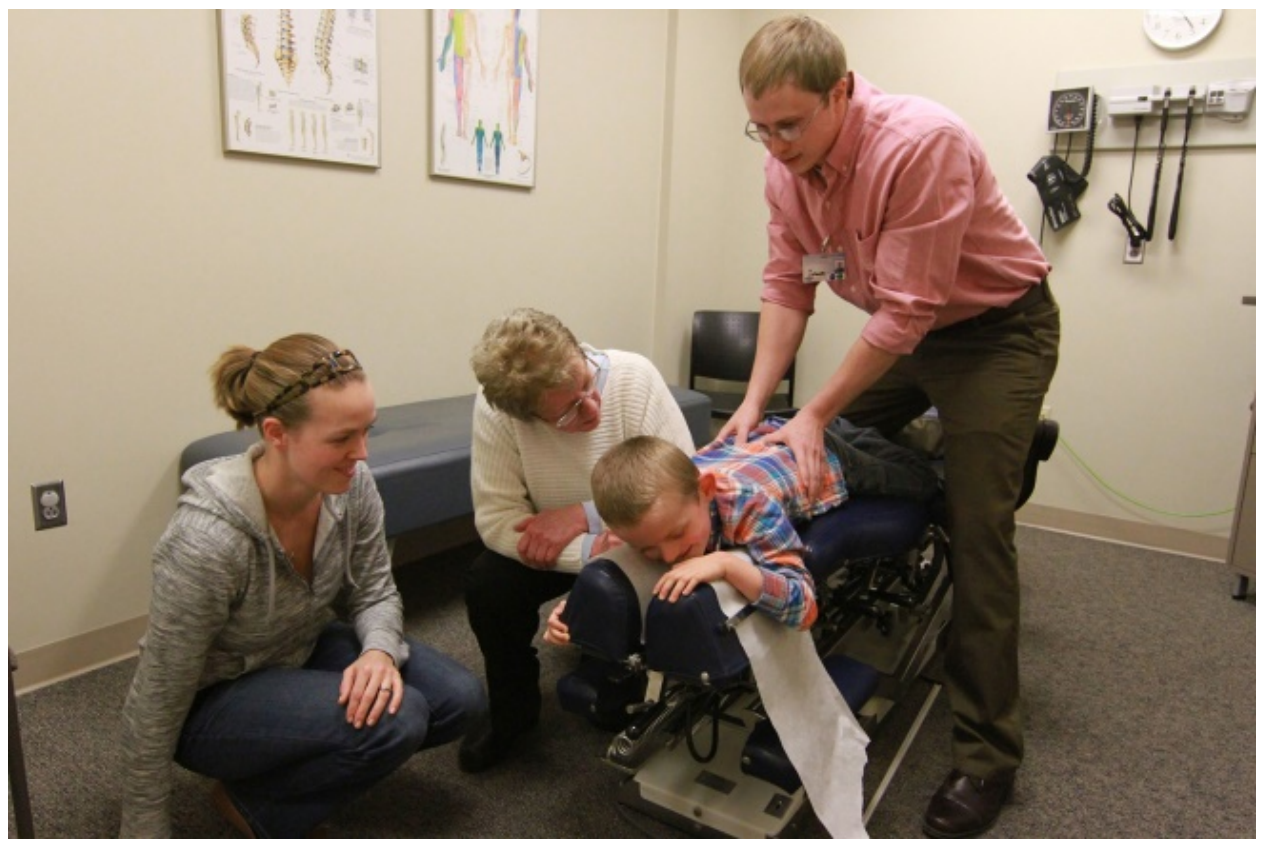

Figure 2. Chiropractic adjustment of the thoracolumbar junction. 
winging in elbow supported prone positioning. While prone the patient's knees were in oblique 90 degree flexure. Patient also had lumbar and thoracic flexion while sitting. The patient was treated using a dynamic neuromuscular stabilization (DNS) protocol (Figure 3) based on patient presentation and infantile behavior and posture. The treating clinician identified the patient was at the developmental level of an eight-week year old child (seen most in the flexed position of his right hand) [9]. Rehabilitative procedures consisted of 4 visits to the clinic's rehab department involving DNS loading exercises bilaterally for 15 minutes. These procedures were concluded with parental education on exercises and request to continue exercises at home.

The patient continued developmental care at school with his OT and PT through an Individualized Education Program (IEP) addressing his needs throughout the chiropractic care plan. He remains under treatment on a monthly basis.

Since the beginning of the treatment 22 weeks ago the child has continued to make significant improvement. The child was provided a stroller along with leg braces to be used at night and ankle braces to be used during the day through his schools IEP. At the time of this writing, the patient can pull himself up and walk extended distance either holding onto the parent's hands or with an ambulatory device.

Mother reports bedtime has become less challenging to go to sleep. Care coupled with dietary changes has improved child's intestinal motility and bowel movements. The patient's demeanor has changed dramatically. The patient presents in a "happy mood", laughing and smiling throughout the visits and attempts to use purposeful language. The parents report the child is more content and "tries to do more physical ac-

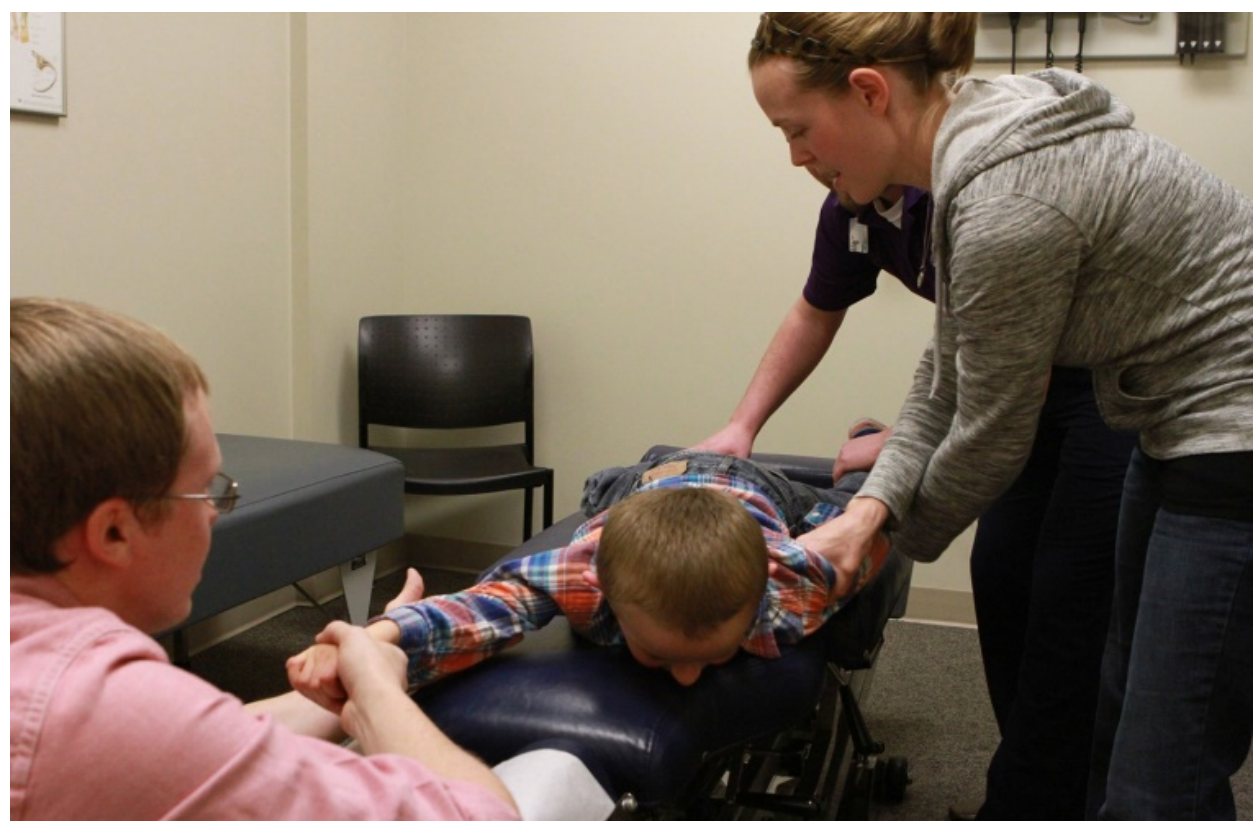

Figure 3. DNS patient positioning and application. Presentation is 4 - 5-month old contra lateral crawl positioning. Light digital pressure (blanching of the fingernail bed) placed upon on the right humerus medial epicondyle in ulnar deviation and the medial SI with bent knee and centration of the left humeral head. 
tivity". The patient appears to want to interact with others. For the first time since the child's birth the family reports being able to go out for a dinner at a restaurant without fear of disruption, which the mother describes as a reward beyond description.

In review of the child's IEP, his educational team recorded "huge improvements this year in the child's ability to stand with assistance and walk with the use of a walker" and noted "increased willingness to verbalize via imitation of sounds has been observed". At the time of this writing, the patient is able to accomplish numerous tasks the child previously had not been able to accomplish at the initial assessment. The patient is able to climb on objects, interact with others children, articulate 1 - 3 words of purposeful language, was hown hands, and wave bye-bye. The child can pull up into an upright position and maintain the posture without assistance. The patient is able to walk with assistance either by gently holding onto the hands of an adult or with the use of a walker. The child has developed the skills of balanced maneuvering of his walker.

\section{Results}

Functional changes were monitored via objective clinic findings, independent clinical examination, with parental and school observation. The patient was very agitated and irritable initially, but improved throughout treatment in attitude and is reported to be in high spirits and a happy child. The parents report the personality has changed drastically from frequently crying to smiling and laughing. The patient improved in quality and quantity of bowel movements which continued throughout care. The parents set their personal expectations for milestone achievements for their son, and they report meeting their goals faster since beginning chiropractic care. The child has been using appropriate topic specific language and more purposeful speech. Currently, the child is ambulating with assistance. The child's positive development and progress both emotionally and functionally has allowed for a more interactive child in the family dynamic.

\section{Discussion}

As addressed earlier the greatest obstacle with development of care options and strategies for AgCC patients lie in the spectrum in the severity and symptoms of disease, making a standard treatment protocol difficult to formulate [10].

In this case study, the patient was diagnosed with a complete agenesis of the corpus callosum. The decision to apply high velocity low amplitude spinal manipulation in conjuncture with DNS exercises was made by both the parents of the patient and the clinician, with an acute awareness there were no comparative expectations to reference.

The treatment protocol was designed to stimulate the brain and activate the somatiosensory pathways. Integration of sensory input received from all parts of the body is largely considered to begin at the level of the spinal cord. Proprioceptive information is shared with the somatosensory cortex through multiple tracts. Three primary pathways arise within the spinal cord. The dorsal column-medial lemniscus system is identified as primarily transmitting sensation of touch, pressure and vibration [11], it is also involved with some conscious appreciation of position and kinesthetic sensations of the 
trunk and extremities [12] [13]. The spinothalamic system transmits pain and thermal sensitivities. The third tract is the spinocerebellar pathway. Information transmitted from this pathway aids in micromanaging active motion through connections on the medial and lateral descending tracts. It also audits somatosensory input for feedback regulation of muscle tone [12] [14].

Within the activation of sensory impulse at the spinal level there is multiple sensory receptors' firing a variety of sensations. Mechanoreceptors responsible for proprioceptive input are located in muscle, tendon, ligament and capsule [12] [13]. Muscle spindles and Golgi tendon organs represent the variation of sensory interpretation generated with mechanoreceptive activation. When these receptors reach threshold and trigger ascending action potentials throughout the somato-sensory pathways the information is registered contralaterally without complete reliance on the corpus callosum. Uncrossed descending cortiocspinal, rubrospinal and recticulospinal tracts will communicate contralaterally in the spinal cord as well. Thus the mechanoreceptor activation/response circuitry will influence the reflexogenic motor responses which inherently influence posture and functional balance [15] [16].

Afferent integration is an essential component of coordinated fluid motor control occurring along all levels of the CNS [12] [13]. Motor function and application rest upon interactions between the supraspinal center, spinal motor networks and peripheral sensory input. Panek et al. found that "low threshold cutaneous mechanoreceptors play critical roles in mediating normal motor function, and ensuring that motor systems adapt to their environment. Furthermore, afferents from these receptors are important in ensuring plastic changes to the central nervous systems microcircuits" [17]. Collectively, the above neurophysiological efforts highlight the human brains neuronal plastic capability, neuromuscular adaptation and functional cortical remapping.

Past studies have identified mechanoreceptive and nociceptive nerve endings residing in the facet capsule in all regions of the spine [18] [19], as well mechanoreceptors in the intervertebral discs [20]. Their presence represents the neurological framework for auditing the proprioceptive and nociceptive input from core motion. Alterations in articular juxtiposion or muscle asymmetries can initiate a maladaptive reflexive change. These changes left unchecked have the ability to perpetuate movement disorders, proprioceptive loss and compensatory motion patterns conducive to pain generation and articular and motor response dysfunction [21] [22] [23] [24] [25]. More recent studies have documented neurophysiological responses to spinal manipulative trust [21] [22] [26]-[32], influencing the mixed nerve action potentials with mechanical stimulation. This stimulation may activate or modulate the somatosensory system and subsequently trigger neuromuscular reflexes. Reflexes believed to inhibit hypertonic musculature, quiet facilitated nociceptive loops and improve spinal function [27]. There has been speculation that spinal manipulative therapy could aid the mechanism responsible "for altering the boundaries of cortical sensory maps, thus, altering the way the central nervous system perceives and processes information" [21].

To evoke neuronal change within the cortex, repeated stimulation over a considera- 
ble time frame would be required to allow proper cognitive/neuronal development. DNS has been utilized throughout Europe and has developed into an effective alternative treatment protocol developing specific motor patterns. DNS was utilized to position the body in a specific position to allow the practitioner to manually stimulate areas/zones of the body by approximating joints to generate a genetically reflexogenic efferent response through the central nervous system. The objective is to facilitate sensory input to address aberrant developmental motor patterns driving cortical assimilation and neurological reorganization [9]. DNS coupled with chiropractic care promote a synergistic stimulation aiding the neuroplastic alteration in the sensory-motor loop initiating leg and upper extremity relaxation and improved motor function.

This experimental treatment protocol was pursued due to the lack of current treatment models for patients with complete AgCC. In this particular case study, the patient could not communicate with the clinicians making it difficult to track cognitive progress and patient perception. Reliance on the parental opinion of the care and objective progress witnessed in the clinic, home and school were utilized in evaluating care effectiveness. The co-management of the patient with PT, OT, and ST at school makes it difficult to precisely quantify chiropractics contributions to this patients improvement margins. In order to determine the success of the chiropractic care in this clinical presentation, more research is needed involving chiropractic inclusion into common treatment methodologies with this and similar clinical conditions.

\section{Limitations}

As with all case reports, this paper reflects promising results of a single patient. Consequently the findings cannot be generalized to a global care strategy.

\section{Conclusion}

This individual case highlighted a treatment option for care of a patient with AgCC. Spinal manipulation in combination with DNS exercises and school therapy classes generated significant improvements in the patient's quality of life. The intention of the study was to provide an alternative care strategy for treatment of AgCC presentations and encourage further investigation of chiropractic care inclusion in similar clinical conditions.

\section{References}

[1] Bénézit, A., Hertz-Pannier, L., Dehaene-Lambertz, G., Monzalvo, K., Germanaud, D., Duclap, D., Guevara, P., Mangin, J.F., Poupon, C., Moutard, M.L. and Dubois, J. (2014) Organising White Matter in a Brain without Corpus Callosum Fibres. Cortex, 63C, 155-171.

[2] Paul, L., Brown, W., Adolph, R., Tyszka, J., Richards, L., Mukherjee, P., et al. (2007) Agenesis of the Corpus Callosum: Genetic, Developmental and Functional Aspects of Connectivity. Nature Reviews Neuroscience, 8, 287-299. http://dx.doi.org/10.1038/nrn2107

[3] Roebuck, T.M., Mattson, S.N. and Riley, E.P. (2002) Interhemispheric Transfer in Children with Heavy Prenatal Alcohol Exposure. Alcoholism: Clinical and Experimental Research, 26, 1863-1871. http://dx.doi.org/10.1111/j.1530-0277.2002.tb02494.x 
[4] Ren, T., Anderson, A., Shen, W.B., Huang, H., Plachez, C., Zhang, J., et al. (2006) Imaging, Anatomical, and Molecular Analysis of Callosal Formation in the Developing Human Fetal Brain. Anatomical Record Part A, Discoveries in Molecular, Cellular, and Evolutionary Biology, 288, 191-204. http://dx.doi.org/10.1002/ar.a.20282

[5] Lavado, A., Ware, M., Paré, J. and Cao, X. (2014) The Tumor Suppressor Nf2 Regulates Corpus Callosum Development by Inhibiting the Transcriptional Coactivator Yap. Development, 141, 4182-4193. http://dx.doi.org/10.1242/dev.111260

[6] Chiappedi, M. and Bejor, M. (2010) Corpus Callosum Agenesis and Rehabilitative Treatment. Italian Journal of Pediatrics, 36, 64. http://dx.doi.org/10.1186/1824-7288-36-64

[7] Erickson, R.L., Paul, L.K. and Brown, W.S. (2014) Verbal Learning and Memory in Agenesis of the Corpus Callosum. Neuropsychologia, 60, 121-130.

http://dx.doi.org/10.1016/j.neuropsychologia.2014.06.003

[8] Tovar-Moll, F., et al. (2015) Structural and Functional Brain Rewiring Clarifies Preserved Interhemispheric Transfer in Humans Born Without the Corpus Callosum. Proceedings of the National Academy of Sciences of the United States of America, 111, 7843-7848. http://dx.doi.org/10.1073/pnas.1400806111

[9] Vojta, V. and Peters, A. (2007) Das Vojta-Prinzip: Muskelspiele in Reflexfortbewegung und motorischer Ontogenese $=$ Flexing Muscles in Reflex Locomotion and Motor Ontogenesis, Springer, Heidelberg.

[10] Klein, R., Hopewell, A., Muniz, D. and Sharieff, A. (2014) B-48 Agenesis of the Corpus Callosum: Context Matters. Cortex, 29, 554.

[11] Donkelaar, H. (2011) The Somatosensory System. In: Donkelaar, H., Ed., Clinical Neuroanatomy, Springer Berlin, Heidelberg, 133-209.

http://dx.doi.org/10.1007/978-3-642-19134-3_4

[12] Riemann, B. and Lephart, S. (2002) The Sensorimotor System, Part 1: The Physiological Basis of Functional Joint Stability. Journal of Athletic Training, 37, 71-79.

[13] Grigg, P. (1994) Peripheral Neural Mechanisms in Proprioception. Journal of Sports Rehabilitation, 3, 2-17. http://dx.doi.org/10.1123/jsr.3.1.2

[14] Ghez, C. (1991) The Cerebellum. In: Kandel, E.R., Schwartz, J.H. and Jessell, T.M., Eds., Principles, Elsevier Science, New York, 627-646.

[15] Francio, V., Boesch, R. and Tunning, M. (2015) Treatment of a Patient with Posterior Cortical Atrophy (PCA) with Chiropractic Manipulation and Dynamic Neuromuscular Stabilization (DNS): A Case Report. The Journal of the Canadian Chiropractic Association, 59, 37-45.

[16] Carrick, F.R. (1997) Changes in Brain Function after Manipulation of the Cervical Spine. Journal of Manipulative and Physiological Therapeutics, 20, 529-545.

[17] Panek, I., Bui, T., Wright, A. and Brownstone, R. (2014) Cutaneous Afferent Regulation of Motor Function. Acta Neurobiologiae Experimentalis, 74,158-171.

[18] McLain, R. and Pickar, J. (1998) Mechanoreceptor Endings in Human Thoracic and Lumbar Facet Joints. Spine, 23,168-173. http://dx.doi.org/10.1097/00007632-199801150-00004

[19] McLain, R.F. (1994) Mechanoreceptor Endings in Human Cervical Facet Joints. Spine, 19, 495-501. http://dx.doi.org/10.1097/00007632-199403000-00001

[20] Roberts, S., Eisenstein, S., Menage, J., Evans, E. and Ashton, I. (1995) Mechanoreceptors in Intervertebral Discs. Morphology, Distribution, and Neuropeptides. Spine, 20, 2645-2651. http://dx.doi.org/10.1097/00007632-199512150-00005

[21] Taylor, H. and Murphy, B. (2010) The Effects of Spinal Manipulation on Central Integra- 
tion of Dual Somatosensory Input Observed after Motor Training: A Crossover Study. Journal of Manipulative and Physiological Therapeutics, 33, 261-272. http://dx.doi.org/10.1016/j.jmpt.2010.03.004

[22] Taylor, H. and Murphy, B. (2010) Altered Central Integration of Dual Somatosensory Input after Cervical Spinal Manipulation. Journal of Manipulative and Physiological Therapeutics, 33, 178-188.

[23] Palmgren, P., Sandstrom, P., Lundqvist, F. and Heikkila, H. (2006) Improvement after Chiropractic Care in Cervicocephalic Kinesthetic Sensibility and Subjective Pain Intensity in Patients with Nontraumatic Chronic Neck Pain. Journal of Manipulative and Physiological Therapeutics, 29, 100-106. http://dx.doi.org/10.1016/j.jmpt.2005.12.002

[24] Panjabi, M.M. (2006) A Hypothesis of Chronic Back Pain: Ligament Subfailure Injuries Lead to Muscle Control Dysfunction. European Spine Journal, 15, 668-676.

http://dx.doi.org/10.1007/s00586-005-0925-3

[25] Budgell, B. (2000) Reflex Effects of Subluxation: The Autonomic Nervous System. Journal of Manipulative and Physiological Therapeutics, 23, 104-106. http://dx.doi.org/10.1016/s0161-4754(00)90076-9

[26] Nougarou, F., Dugas, C., Deslauriers, C., Pagé, I. and Descarreaux, M. (2013) Physiological Responses to Spinal Manipulation Therapy: Investigation of the Relationship between Electromyographic Responses and Peak Force. Journal of Manipulative and Physiological Therapeutics, 36, 557-563.

[27] Colloca, C. and Hinrichs, R. (2005) The Biomechanical and Clinical Significance of the Lumbar Erector Spinae Flexion-Relaxation Phenomenon: A Review of Literature. Journal of Manipulativeand Physiological Therapeutics, 28, 623-631. http://dx.doi.org/10.1016/j.jmpt.2005.08.005

[28] Colloca, C., Keller, T. and Gunzburg, R. (2004) Biomechanical and Neurophysiological Responses to Spinal Manipulation in Patients with Lumbar Radiculopathy. Journal of Manipulative and Physiological Therapeutics, 27, 1-15. http://dx.doi.org/10.1016/j.jmpt.2003.11.021

[29] Colloca, C., Keller, T. and Gunzburg, R. (2003) Neuromechanical Characterization of in Vivo Lumbar Spinal Manipulation. Part II. Neurophysiological Response. Journal of Manipulative and Physiological Therapeutics, 26, 579-589.

[30] Wood, T., Colloca, C. and Matthews, R. (2001) A Pilot Randomized Clinical Trial on the Relative Effect of Instrumental (MFMA) versus Manual (HVLA) Manipulation in the Treatment of Cervical Spine Dysfunction. Journal of Manipulative and Physiological Therapeutics, $24,260-271$.

[31] Colloca, C. and Keller, T. (2001) Electromyographic Reflex Responses to Mechanical Force, Manually Assisted Spinal Manipulative Therapy. Spine, 26, 1117-1124. http://dx.doi.org/10.1097/00007632-200105150-00005

[32] Colloca, C., Keller, T., Gunzburg, R., Vandeputte, K. and Fuhr, A.W. (2000) Neurophysiologic Response to Intraoperative Lumbosacral Spinal Manipulation. Journal of Manipulative and Physiological Therapeutics, 23, 447-457.

http://dx.doi.org/10.1067/mmt.2000.108822 
Submit or recommend next manuscript to SCIRP and we will provide best service for you:

Accepting pre-submission inquiries through Email, Facebook, LinkedIn, Twitter, etc. A wide selection of journals (inclusive of 9 subjects, more than 200 journals)

Providing 24-hour high-quality service

User-friendly online submission system

Fair and swift peer-review system

Efficient typesetting and proofreading procedure

Display of the result of downloads and visits, as well as the number of cited articles

Maximum dissemination of your research work

Submit your manuscript at: http://papersubmission.scirp.org/

Or contact jbbs@scirp.org 